\title{
Saúde Mental na Atenção Básica: um estudo epidemiológico baseado no enfoeue de risco
}

\author{
Mental Health in the Basic Attention: an epidemic study based on the risk focus
}

Salud Mental en la Atención Básica: un estudio epidemiologico basado en el enfoque de riesgo

\author{
Fábia Barbosa de Andrade', Alessandra Isis Cirne Bezerra', Ana Lúcia Félix de Pontes", \\ Maria Oliveira Ferreira Filha', Rodrigo Pinheiro de Toledo Vianna'II', Maria Djair Dias', Antônia Oliveira Silva' \\ 'Universidade Federal da Paraíba. Programa de Pós-Graduação em Enfermagem. João Pessoa, PB \\ "Universidade Federal da Paraíba. Hospital Lauro Wanderley. João Pessoa, PB \\ "I'Universidade Federal da Paraíba. Departamento de Nutrição. Ioão Pessoa, PB
}

Submissão: $04 / 05 / 2009$

Aprovação: $31 / 08 / 2009$

\section{RESUMO}

As transformações de cunho social-político e econômico ocorrido nas últimas décadas repercutem em todos os setores da sociedade. Fato Que traz transformações para a saúde mental da população. Teve como objetivo realizar a população com risco para adoecimento mental. Trata-se de um estudo epidemiológico de corte transversal realizado em uma comunidade de loão Pessoa-PB, utilizando-se Self Report Questionnaire-20 (SRQ-20). Observou-se associação do Risco para Depressão e Ansiedade com o sexo feminino (59,40\%), faixa etária de 40 a 65 anos (66\%), ocupação de serviços domésticos (65,5\%). Somos levados a reconhecer Que o sofrimento mental tem crescido relativamente na amostra estudada e a partir dessa constatação faz-se necessário uma atenção imediata aos problemas de saúde mental nas pessoas da comunidade.

Descritores: Saúde mental; Atenção primária à saúde; Epidemiologia; Grupos de risco.

\begin{abstract}
The transformations of stamp social, political and economical happened in the last decades they rebound significantly in all the sections of the society. And if it cannot leave of considering the influences of those transformations for the mental health of the population. It is a epidemic cohort study that aimed at accomplishing the risk for mental disease in the population assisted by the Unit of Health of the Family of the habitational group, neighborhood of Mangabeira, city of João Pessoa-PB, where Self Report Questionnaire-20 was used (SRQ-20). Association of the Risk was observed for Depression and Anxiety with the feminine sex (59,40\%), among the age group from 40 to 65 years $(66 \%)$, in the occupation of domestic services $(65,5 \%)$. We are mischievous to recognize that the mental suffering has been growing relatively in the studied sample and starting from that verification it is done necessary an immediate attention to the problems of mental health in the community's people.
\end{abstract}

Descriptors: Mental health; Primary health care; Epidemiology; Risk groups.

\section{RESUMEN}

Las transformaciones de carácter social, político y económico ocurridas en las últimas décadas repercuten en todos los sectores de la sociedad. Y no se pueden dejar de considerar las transformaciones ejercen en la salud mental de la población. Con el objetivo de investigar el risco para enfermedad mental en una comunidad de João Pessoa, se trata del un estudio de tipo epidemiológico de corte transversal, donde se aplicó el Self Report Questionnaire-20 (SRQ-20). Se observó asociación de Riesgo para Depresión y Ansiedad con el sexo femenino (59,40\%), en el intervalo de edad de 40 a 65 años (66\%), en la ocupación de servicios domésticos (65,5. Somos llevados a reconocer Que el sufrimiento mental creció relativamente en la muestra estudiada e a partir de esta constatación se hace necesaria una atención inmediata a los problemas de salud mental de las personas de la comunidad.

Descriptores: Salud mental; Atención primaria en salud; Epidemiología; Grupos de riesgo. 


\section{INTRODUÇÃO}

As tendências em matéria de análise da atenção à saúde mental têm variado conforme as épocas e os distintos contextos. Elas têm estado Quase unilateralmente definidas em termos técnicos sanitários, excluindo determinantes significativos tais como: sócioeconômicos, políticos, culturais e religiosos.

De todos os males vividos pelo o homem, a loucura, a doença mental e o sofrimento psíquico e emocional, parecem atingir indistintamente pessoas de Qualeuer nacionalidade, raça, classe social e religião. Contudo, sabemos Que os mais pobres são os Que mais padecem pela falta de atenção e cuidado.

O Relatório sobre a saúde no mundo, revela Que, no ano 2000 os transtornos mentais e neurológicos foram responsáveis por $12 \%$ do total de anos vida ajustados por incapacitação (AVAI), perdidos em virtude das doenças incapacitantes (transtornos depressivos, a esquizofrenia, os resultantes do abuso de substâncias, a epilepsia, o retardo mental, os transtornos da infância e adolescência e a doença de Alzheimer), e Que até o ano 2020, a carga dessas doenças terá crescido para 15\%, sendo Que apenas uma minoria recebe tratamento adequado. Nesse documento, são descritos, além dos transtornos comuns Que geralmente causam incapacitação grave, os principais fatores Que determinam esta prevalência, a manifestação e o curso desses transtornos, tais como a pobreza, o gênero, a idade, os conflitos e catástrofes, doenças físicas graves e o clima familiar e social ${ }^{(1)}$.

Com a implantação do Programa de Saúde da Família, desde 1996, na cidade de João Pessoa/PB, ampliou-se a rede de cuidados primários de saúde. Mas, verificou-se Que $90 \%$ dos profissionais das equipes de saúde da família ainda não se sentem preparadas para atender a demanda de pessoas com sofrimento psíquico e além do mais, desconhecem modos de abordagem a essa clientela, no sentido de ajudá-los a superar seus problemas e conseeüentemente melhorar a saúde emocional e a qualidade de vida $^{(2)}$.

Estimando-se Que os transtornos mentais estão presentes em cerca de $12 \%$ da população, nesse estudo, pretendemos inicialmente identificar: Quem são as pessoas com risco para adoecimento mental na população da comunidade Ambulante do Conjunto habitacional Mangabeira IV, no Bairro de Mangabeira? Quais as situações Que geram sofrimento emocional e favorecem o aparecimento de risco para transtornos mentais? Quais as estratégias Que podem ser viabilizadas pela equipe de saúde da família para diminuir os riscos à saúde mental da população?

O risco é definido pela presença de um ou de uma associação de critérios, uns de ordem médica, outras de ordem social. Já os fatores de risco relacionados a aspectos comuns da vida cotidiana como saúde, salário, estado civil, idade, escolaridade, empregos, falta de ocupação, tipo de profissão, podem funcionar como dispositivos Que visam asseguram uma detecção sistemática e rápida de certos riscos ${ }^{(3)}$.

O sofrimento emocional é revelado através de Queixas clínicas, alguns de origem orgânica outros de fundo psíquico, mas Que revelam o impacto das variáveis contextuais na vida dos indivíduos.

A explicação conclusiva do informe da saúde mental, não obstante, é que os transtornos neuropsiquiátricos propriamente ditos são responsáveis por uma fração dos problemas determinados pela morbidade social e psicológica. O alcoolismo, a drogadição, o suicídio e as tentativas de suicídio, a violência contra a mulher, o maltrato e abandono das crianças, a prostituição forçada, os delitos, a violência, entre outros constituem o conjunto de situações existentes na sociedade Que provocam sofrimento mental na população(4).

A importância de conhecer as taxas dos transtornos mentais na população, parte da certeza de um melhor planejamento dos serviços de saúde oferecidos à comunidade. Além disso, a identificação dos fatores de risco ou de proteção associados ao surgimento dos principais transtornos pode funcionar como uma base de informação para a indicação de tratamentos e principalmente para elaboração dos programas de prevenção, bem como na organização e implementação da rede de serviços de saúde mental ${ }^{(5)}$.

Desse modo, o presente estudo teve os seguintes objetivos: descrever o perfil sócio demográfico das pessoas com risco para adoecimento mental; identificar situações de risco para a saúde mental e correlacionar com o sofrimento emocional; estudar a influência dos fatores de risco para a saúde mental no desencadeamento do adoecimento mental na população estudada; descrever estratégias Que podem ser viabilizadas pela equipe de saúde da família para diminuir os riscos para o adoecimento mental.

\section{METODOLOGIA}

Trata-se de um estudo epidemiológico de corte transversal onde foram investigadas as características e condições de vida da população atendida em uma Unidade de Saúde da Família. A lógica de base da moderna epidemiologia estrutura-se em torno do conceito fundamental - risco - e de um conceito correlato - fator de risco $^{(5)}$.

O cenário da peseuisa foi a comunidade coberta pela Unidade de Saúde da Família Mangabeira IV Ambulantes, localizada na micro-região sul do município de João Pessoa, bairro de Mangabeira IV, Estado da Paraíba.

Fizeram parte da mostra $18 \mathrm{I}$ pessoas. O tamanho da amostra foi calculado para obter uma representatividade da população da USF considerando a prevalência do risco de Depressão nesta população de $50 \%$ e precisão de $5 \%$ considerando um intervalo de confiança de $90 \%$. Para estes parâmetros o número da amostra foi de 163 pessoas.

A seleção da amostra ocorreu de forma aleatória, contudo considerou-se como critério de inclusão, ter idade acima de 15 anos e ser cadastrado na Unidade de Saúde da Família e aceitar participar da pesquisa. O critério de inclusão da idade acima de 15 anos justifica-se através dos estudos da OPAS, Que confirmam ser a faixa etária de 15 a 44 anos, na Qual os transtornos mentais estão mais presentes, sendo responsáveis pelo ônus da incapacidade $^{(1)}$.

Adotou-se como critério de exclusão da amostra pessoas com deficiência mental e com transtorno mental severo, devido a prejuízos apresentados na área da linguagem e da cognição dificultando a compreensão das Questões e a conseQüente resposta às mesmas.

Para coleta de dados foram utilizados dois Questionários: o primeiro, um Questionário de sintomas denominado Self Report Questionnaire-20 (SRQ-20) Que possui 20 Questões relativas ao 
período do mês anterior à entrevista. O SRQ-20 foi recomendado pela Organização Mundial da Saúde para estudos comunitários e em atenção primária à saúde. Esse instrumento foi validado no Brasil $^{(6)}$, onde constam dados de identificação e levantamento de sintomas apresentados pelo indivíduo nos últimos trinta dias. Tal instrumento permite avaliar o(s) risco(s) para adoecimento mental, Que se constitui em Risco para Depressão e Ansiedade. Foi utilizado ainda um outro Questionário sobre os fatores de risco, também relacionados com os últimos 30 dias, o Qual contempla situações cotidianas da vida como sofrimento; violência; convivência com usuários de álcool e/ou drogas; desemprego; abandono; migração; doenças, rompimentos e falecimento de familiares, entre outros.

Concluída a coleta de dados, as informações foram armazenadas em um banco de dados através da utilização do software Statistical Package for Social Sciences (SPSS) 8.0 for Windows.

Foi feita a caracterização da amostra considerando-se as variáveis sócio-demográficas; as freeüências dos riscos (análise univariada) para adoecimento mental: Risco para Depressão e Ansiedade. Além dos cruzamentos dos riscos com as variáveis sócio-demográficas (sexo, estado civil, idade, ocupação) e com os fatores de risco (análise bivariada). Utilizou-se o teste estatístico qui-Quadrado e a probabilidade menor Que 10\% para rejeição da hipótese de não associação.

Esta pesquisa foi submetida ao Comitê de Ética do CCS e obteve parecer favorável a execução da mesma, respeitando-se a Resolução 196/96 do Conselho Nacional de Saúde (CNS).

\section{RESULTADOS E DISCUSSÃO}

A apuração da amostra se deu em torno de 181 entrevistados, dando-se subsídios para que se possa identificar que a maioria da amostra foi formada por mulheres $(85,6 \%)$; faixa etária entre 40 a 65 anos (53,6\%); estado civil casado (55,2\%); ensino fundamental incompleto (63\%); com ocupação Do lar $(58,6 \%)$; religião católica (70,7\%); situação empregatícia desempregada $(49,7 \%)$ e não possuíam nenhuma renda $(49,7 \%)$.

Contudo os dados apresentados pelo Sistema de Informação da Atenção Básica mostram Que nesta área de abrangência da USF Ambulantes, a população é $54,1 \%$ do sexo feminino e $45,9 \%$ do sexo masculino; $86,2 \%$ são alfabetizados e que a renda familiar é em torno de dois a três salários mínimos. As ocupações mais referidas são do lar, estudantes, aposentados, vendedores ambulantes, domésticas e sem ocupação.

As mulheres as Que mais freqüentam os serviços de saúde, principalmente porQue culturalmente são responsáveis pelo cuidado com a saúde da família e também porQue há ações específicas para o cuidado com a saúde da mulher. Vê-se Que a maioria dos homens está ausente dos serviços de saúde. Os motivos para tal podem ser de natureza cultural, econômica ou educacional.

Embora já existam mudanças de comportamento entre os homens na sociedade, culturalmente o cuidado com a família está ao encargo da mulher e os serviços não dispõem de ações de saúde específicas para o grupo masculino. Os serviços não funcionam em horário noturno, o Que dificulta o acesso dos Que trabalham no horário diurno. Por outro lado, sabe-se Que entre os homens predominam problemas como o consumo de álcool e drogas e também a violência. Contudo, nestes serviços inexistem ações estratégicas por parte da equipe de saúde no sentido de enfrentar tais problemas.

Alguns dos dados sócio-demográficos foram correlacionados ao Risco para adoecimento mental: sexo, estado civil, faixa etária e ocupação. $\mathrm{O}$ Que se esconde atrás da deficiência não é a irrupção do patológico, mas o reino da desigualdade adeuirida na luta pela vida concebida como um percurso de obstáculos Que mede sempre uma inferioridade ${ }^{(3)}$.

Observou-se Que aqueles Que referiram sintomas associados ao risco para Depressão e Ansiedade 59,40\% são mulheres e 26,90\% são homens. O Qui-Quadrado para essa associação teve um valor de $p=0,02$, portanto significativa a associação entre gênero e risco para Depressão e Ansiedade, revelado pelo Quantitativo de mulheres maior Que o de homens, de modo Que estas estão mais vulneráveis a este risco. Assim, considerando a variável "sexo" no presente estudo verificou-se Que foram as mulheres Que mais referiram os sintomas para o adoecimento mental. As mulheres são mais susceptíveis aos distúrbios reativos, afetivos, psicossomáticos e psicoses maníaco-depressivos; os dados, segundo o autor, a respeito do sexo mostram a relação entre doença mental e o papel social: ao homem - o trabalho e o mando; à mulher - a família e as emoções $^{(7)}$.

Desse modo, os dados nos revelaram que $66 \%$ dos entrevistados se encontravam na faixa etária de 40 a 65 anos; 55,2\% acima de 65 anos e $34,5 \%$ estavam na faixa entre 21 a 40 anos. O teste de Qui-Quadrado para esse dado teve um valor de $p=0,01$, sendo portanto significativa a associação entre as faixas etárias e o risco para Depressão e Ansiedade, revelado através do Quantitativo das variáveis referentes a faixa etária de 40 a 65 anos por apresentar um percentual de $66 \%$, e na faixa etária acima de 65 anos $(55,2 \%)$, contudo no que diz respeito a faixa etária 21 a 40 anos não houve associação visto se apresentou um percentual de $34,5 \%$.

No tocante a variável idade, utilizou-se no presente estudo os estágios da personalidade de Erik Erickson, onde se percebeu Que a maioria das pessoas se encontrava no estágio 7 e 8 . No primeiro (estágio 7) por volta de 40 a 65 anos, as pessoas de meia idade mostram uma tendência mais elevada para o desenvolvimento de depressões do Que nos adultos mais jovens, Que pode estar relacionado às decepções da vida e às expectativas frustradas Quando revisam o passado. Já no estágio 8 os transtornos da ansiedade são mais comuns, talvez por haver uma certa revisão de sua própria história vital, declínio das funções físicas, pode contribuir para o surgimento de doenças psicossomáticas como a hipocondria e a depressão. Cada idade possui suas possibilidades patológicas específicas, pois cada uma apresenta modificações somáticas próprias e se inscreve em um papel social determinado ${ }^{(7)}$.

No Que diz respeito à associação das variáveis risco para depressão e ansiedade com a ocupação, os resultados evidenciaram Que predominava pessoas com as seguintes ocupações: $65,5 \%$ se encontravam nos serviços domésticos, Que compreendia as mulheres Do lar e aQuelas Que desempenhavam atividades domésticas; 39,3\% estavam trabalhando no serviço terciário e $34,8 \%$ estavam sem ocupação (ou seja desempregado). $28,6 \%$, se enQuadravam na categoria outros, Que se refere a serviços tais como: estudantes, agricultores, trabalhadores de couro, pedreiro, ambulante, entre outros. O Qui-Quadrado para essa associação teve um $p=0,01$, sendo dessa forma significativa a associação entre a ocupação Do lar e o risco para Depressão e Ansiedade por haver apresentado 
um percentual de 65,5\%; as demais categorias não apresentaram associação, uma vez Que não foi observado um Quantitativo elevado de entrevistados Que apresentaram o referido risco. Na ocupação, observou-se Que os riscos para adoecimento se fizeram mais presentes nas mulheres Que no período do estudo possuíam a ocupação de serviços domésticos (cuja constituição se deu com as mulheres Do lar e aquelas que são Domésticas). A dificuldade existente entre os problemas do trabalho e a capacidade de produção representam as mais importantes áreas de tensão social de nossa existência ${ }^{(7)}$.

Com relação à correlação de variáveis: risco pra depressão e ansiedade e estado civil, mostrou que $51 \%$ possuíam o estado civil casado; $52,2 \%$ são solteiros e $68,6 \%$ possuem outro tipo de união: separados ou viúvos. O Qui-Quadrado encontrado foi de $p=0,18$, não sendo portanto significativo, uma vez Que revelou um valor de p > 10\%, e por sua vez não foi possível observarmos associação nas categorias do estado civil, entre os casados (5l\%), solteiros $(52,2 \%)$, bem como na maior categoria em percentual de separados e viúvos $(68,6 \%)$. Considerando o risco para o adoecimento mental Quanto ao estado civil, percebeu-se Que se fizeram mais presentes na categoria outros, Que no presente estudo diz respeito aos viúvos e separados. É importante considerar a presença do companheiro como fator de risco para adoecimento, pois a dinâmica familiar é algo imprescindível para compreensão do ser humano enQuanto elemento social, pois é no meio familiar Que ele empreende sua constituição individual, o desenvolvimento psicológico e sua personalidade e a organização de sua identidade.

No tocante à associação do risco para depressão e ansiedade e o fator de risco existência de alguma situação de sofrimento, o teste Qui-Quadrado revelou um valor de $p=0,00$, sendo portanto significativa a associação entre ambas as variáveis, uma vez Que 60,6\% das pessoas Que apresentaram o risco para Depressão e Ansiedade afirmaram o referido fator de risco, frente a $28 \%$ Que embora referissem o fator de risco não apresentassem o risco para adoecimento, podendo-se inferir que as pessoas Que convivem com o sofrimento cotidiano são mais vulneráveis aos danos à saúde mental.

No Que se refere ao fator de risco existência de alguma prática ou sofrimento de violência, foi observado Que o teste de QuiQuadrado revelou um valor de $p=0,02$, sendo portanto significativa a associação entre o fator de risco acima e o risco para Depressão e Ansiedade, visto Que 15,2\% das pessoas Que apresentaram o risco para Depressão e Ansiedade afirmaram a existência desse fator de risco (mostrando um percentual maior), frente a 4,9\% Que embora não apresentassem o risco para adoecimento mental afirmaram a presença do fator de risco.

No Que se refere à situação das pessoas conviverem com alguém Que usa álcool ou drogas, foi encontrado um valor de $p=0,07$, sendo portanto significativa, o Que vem nos mostrar Que não foi observada associação. A variável convivência com alguém Que usa álcool ou drogas, não foi considerada fator de risco, mas sim fator de proteção, uma vez $21,2 \%$ das pessoas Que apresentaram risco para Depressão e Ansiedade afirmaram a presença desse fator de risco, frente a 31 ,7\% (maior percentual) Que embora afirmassem o referido fator não apresentou o risco par adoecimento mental.

No Que diz respeito ao fator de risco existência de alguém desempregado relacionado com o risco para Depressão e Ansiedade, foi obtido um valor de $p=0,31$, sendo portanto não significativo, por haver apresentado um valor de $p>10 \%$, bem como por observarmos Que não há uma diferença significativa entre os Que apresentaram o risco para adoecimento mental e o referido fator de risco $(59,6 \%)$ e aQueles Que não apresentaram o risco para Depressão e Ansiedade mas referiram o fator de risco $(54,9 \%)$.

No Que se refere ao fator de risco abandono, observou-se que o valor do teste Qui-Quadrado foi de $p=0,00$, sendo portanto significativa a associação entre o fator de risco e o risco para Depressão e Ansiedade, uma vez Que podemos verificar Que o maior percentual foi encontrado naQueles Que apresentaram o referido risco para adoecimento mental e o fator de risco abandono $(37,4 \%)$ frente a $13,4 \%$ Que embora afirmassem a presença desse fator de risco em suas vidas não apresentaram o risco para Depressão e Ansiedade.

Quanto à existência do fator de risco migração cruzado com a variável risco para Depressão e Ansiedade foi observado um valor de $p=0,38$ não sendo significativa a associação entre o fator de risco migração e Risco para Depressão e Ansiedade, por ter se apresentado um $p>10 \%$, bem como pelo fato do Quantitativo, cujos valores se apresentaram próximos, entre os Que migraram e apresentaram risco para Depressão e Ansiedade (16,2\%) e aqueles Que embora afirmassem esse fator de risco não apresentaram o referido risco para adoecimento mental $(13,4 \%)$.

Quanto à existência de algum parente com doença grave, foi obtido um valor de $p=0,05$ sendo portanto significativa a associação entre o referido fator de risco e o risco para Depressão e Ansiedade, uma vez que o Quantitativo daqueles Que referiram o fator de risco e apresentaram o risco para Depressão e Ansiedade se mostrou maior $(39,4 \%)$ do Que aQueles Que não apresentaram o referido risco para adoecimento mental, mas referiram o fator de risco $(20,7 \%)$.

No tocante à existência de algum tipo de rompimento na família, observou-se um valor de Qui-Quadrado de $p=0,08$, sendo portanto significativo a associação entre o fator de risco acima e o risco para Depressão e Ansiedade, uma vez Que 29,3\% daQueles Que afirmaram o referido fator de risco também apresentaram o risco para Depressão e Ansiedade, frente a 19,5\% Que embora afirmassem o fator de risco não apresentaram o risco para Depressão e Ansiedade.

Quanto presença do fator de risco morte de alguém estimado, foi obtido um valor de Qui-Quadrado de $p=0,00$, sendo mostrando um dado significativo, o Que nos mostra Que existe associação entre o fator de risco (falecimento de alguém Querido) e o risco para depressão e ansiedade, uma vez Que o percentual daqueles Que apresentaram o risco para Depressão e Ansiedade e o fator de risco foi maior $(41,4 \%)$ do Que aqueles Que não apresentaram o referido risco para adoecimento mental embora referissem o fator de risco $(23,2 \%)$.

No Que se refere à existência de outra(s) situação(ões) Que estavam Ihe trazendo sofrimento emocional, verificou-se que o valor de Qui-Quadrado obtido foi de $p=0,07$, sendo portanto significativa a associação este fator de risco e o risco para Depressão e Ansiedade, visto Que o Quantitativo daqueles Que apresentaram o risco para adoecimento mental e o fator de risco foi maior $(49,5 \%)$ do Que aQueles Que não apresentaram o risco mas referiram o fator de risco $(37,8 \%)$. Os comentários mais verbalizados pelos entrevistados foram situações de desemprego, problemas 
financeiros, familiares e de saúde, entre outros. Os transtornos depressivos são as afecções neuropsiquiátricas mais comuns (representam um Quinto do peso de morbidade nas mulheres e um sétimo nos homens). Estes transtornos constituem a maior proporção do peso de morbidade Que suporta a comunidade e Quando seu numero se agrega aos transtornos de ansiedade, são responsáveis por entre um terço e um Quarto do total de consultas realizadas no nível primário da atenção, segundo documentam diferentes estudos ${ }^{(4)}$.

Existe uma progressiva incorporação de um conjunto de fatores patogênicos Que foram agrupados sob a rubrica de 'estresse social', contudo, esta categoria não proporciona operações satisfatórias tanto na clínica como na epidemiologia. Mas, é impossível negar as vicissitudes próprias das sociedades modernas como aglomerações, isolamento, trabalho entediante e/ou insatisfatório, desemprego, entre outros, Que se constituem em importantes fatores responsáveis pelo adoecimento no campo dos distúrbios mentais. Não faltam contingências na vida cotidiana para Que as pessoas experimentem frustrações, conflitos, incerteza e insegurança, Que por sua vez estão relacionados com a ocorrência de um grande número de doenças ${ }^{(9)}$.

Após a seleção e análise dos fatores vinculados ao risco de adoecimento da população, o próximo passo Que surge diz respeito à elaboração de um programa específico, onde o conteúdo das ações vai variar Qualitativamente e Quantitativamente em todos os componentes, os Quais vão depender daqueles fatores de risco Que foram identificados e selecionados e Que passarão a ser o objeto de uma ação de controle oportuno, de acordo com as estratégias de intervenção dirigidas a sua erradicação, redução de sua intensidade ou freqüência nas pessoas ou grupos ${ }^{(10)}$.

A elaboração de estratégias de saúde, construída conforme o paradigma do risco, perpassa por dois pontos: 1) seleção do tipo de estratégia de intervenção Que será utilizada (e isso vai depender da natureza dos fatores de risco, da capacidade do setor de mobilizar a sociedade para controlá-los, bem como a visão de saúde existente); 2) especificação dos elementos constitutivos de programa de aplicação da estratégia definida ${ }^{(10)}$.

O trabalho também é interação social, as relações sociais entre os sujeitos, no momento do planejamento, podendo revelar a racionalidade Que os mesmos imprimem às suas ações-Que reage ao que propõe, a fim de obter êxito (...), mediante cooperação ou conflito, em busca do êxito, estabelecendo um diálogo com o outro para compartilhar uma interpretação sobre a situação em foco, e, desse modo, construir por meio do consenso um propósito comum, mediante atos de fala sustentados por pretensões de validade.

Desse modo, interessados em proporcionar esse espaço de troca de experiências, através da partilha de problemas e por sua vez desenvolvendo estratégias de promoção da Saúde Mental e de prevenção de transtornos mentais, buscou-se na Terapia Comunitária, atividade Que vem sendo desenvolvida há mais de 19 anos no Ceará, uma nova estratégia de integralidade, Que se baseia na troca de experiências e vivências da comunidade, a fim de nutrir a autonomia dos participantes, através do rompimento da concentração de informações tidas pelos profissionais, fazendo com Que estes conhecimentos circulem entre todos, para Que dessa forma se beneficiem, deixando este de ser limitado para ser compartilhado de forma horizontal e circular, onde cada um se torna terapeuta de si mesmo, a partir da escuta das histórias de vida. Todos são co-responsáveis na busca de soluções e superação dos desafios do cotidiano ${ }^{(11)}$.

Entrar na cultura relacional é abordar uma paisagem social de conflitos, munida da única certeza de ter perpetuamente Que retecer a imagem frágil de uma sociabilidade perfeita, cujos fragmentos estão exibidos no universo unidimensional do psicológico. Desse modo, trabalhar a cultura vem se apresentando como alternativa de uma situação em Que os investimentos sociais se eximem e as alternativas políticas estão embaralhadas ${ }^{(3)}$.

Desse modo, e de posse dos resultados acima apresentados entende-se como é importante a participação das USFs do município de João Pessoa, no levantamento desses indicadores de saúde mental, não só pelo fato do Programa de Saúde da Família se constituir em uma estratégia no modelo de atenção, mas também, por acreditar Que participar dessa inclusão na saúde mental é trabalhar na perspectiva da Reforma Psieuiátrica, Que vem a cada dia procurando reconstruir a visão do homem, não apenas como ser biológico, mas acima de tudo como um ser social, entendido e inserido em sua realidade contextual.

\section{CONSIDERAÇÕES FINAIS}

As ações de saúde mental durante muito tempo foram consideradas de modo isolado onde se buscava a promoção dessas ações a partir da exclusão de fatores sociais, econômicos, culturais, políticos, entre outros. Desse modo, a compreensão no âmbito da saúde mental passa pelo crivo desses conceitos, a fim de percebermos como o desemprego, o aumento da pobreza, o abandono, a desesperança, o isolamento social, entre outras situações, afetam a Qualidade vida das pessoas.

Durante a execução do presente estudo, no Qual foi trabalhado com um eixo temático de risco para adoecimento mental na população atendida na atenção básica (Unidade de Saúde da Família), no tocante ao enfoque epidemiológico de risco e seu conceito correlato de fatores de risco, foi um tanto Quanto desafiador por vários aspectos: primeiro compreender a complexidade das relações do indivíduo em sua realidade contextual, segundo a prudência de selecionar, em meio à escassez de material literário a respeito do sofrimento mental no âmbito da epidemiologia, principalmente, a compreensão dessa relação após o cruzamento das variáveis envolvidas no estudo.

Quanto à análise bivariada das Questões trabalhadas entre os riscos x fatores de riscos, no Que se refere ao risco para Depressão e Ansiedade, percebeu-se Que houve associação do fator de risco existência de alguma situação de sofrimento; prática ou sofrimento de violência; sentimento de abandono; parente com doença grave; algum rompimento na família; falecimento de alguém estimado e outras situações difíceis Que estejam vivenciando.

Outro aspecto que merece serem mencionado, considerando a importância e a necessidade de estudos populacionais posteriores acerca do tema de risco para adoecimento mental, diz respeito ao instrumento Que foi utilizado no presente estudo, de modo que algumas considerações merecem ser esclarecidas.

Os dados acima revelam a grande necessidade de ações de saúde mental na atenção básica, pois percebemos as próprias mudanças sociais, políticas, econômicas, entre outras, e como essas mudanças 
influenciam significativamente nas esferas biológica e mental. Esses dados nos levam a compreensão do homem, de fato, enQuanto ser social e compreendido em seu contexto histórico.

Deixar de considerar os fatores envolvidos no conceito de saúde mental é continuar lidando com o modelo de saúde pública, ainda de modo insipiente. O maior desafio, talvez, esteja atrelado à desconstrução do modelo psiQuiátrico Que durante muito tempo vigorou e Que ainda teima em se manter erguido. Daí surge a necessidade de aprender a trabalhar a cultura relacional na comunidade, a fim de amenizar o sofrimento mental daqueles que procuram os serviços de saúde muitas vezes necessitando não só de abordagem biológica mas acima de tudo de sentirem escutados no que se refere aos seus medos, angústias, tristezas, entre outros.

Esperamos Que este estudo possa oferecer subsídios para a construção de posteriores trabalhados na área da epidemiologia em saúde mental, bem como sensibilize os gestores de saúde e os profissionais Que se encontram nos serviços da atenção básica de saúde e Que estes por sua vez, possam elaborar um plano estratégico, onde as ações de saúde mental compreendam a complexidade do processo saúde-doença mental e com isso promovam ações mais amplas Que tragam a melhoria da Qualidade de vida da população humana.

\section{REFERÊNCIAS}

1. Organização Mundial de Saúde. Relatório sobre a saúde no mundo 200 I. Saúde mental: nova concepção, nova esperança. Genebra: Organização Mundial da Saúde; 2001.

2. Carvalho AOD. Saúde Mental: um desafio para o Programa de Saúde da Família [monografia]. João Pessoa: Curso de Enfermagem, Universidade Federal da Paraíba; 2002.

3. Castel R. A gestão dos riscos: da antipsiquiatria à Póspsicanálise. Rio de Janeiro: Francisco Alves; 1987.

4. Desjarlais R, Eisenberg L, Good B, Kleiman A. Salud Mental en el mundo: problemas y prioridades en poblaciones de bajos ingresos. Washington: Organización Panamericana de la Salud; 1997.

5. Fleitlich BW, Goodman R. Epidemiologia. I Bras Psiquiatria 2000; 22(supl II).

6. Mari J, Willians PA. A validity study of a psychiatric screening Questionnaire (SRQ-20) in primary care in the city of São Paulo.

Brit I Psychiatry 1896; 148: 23-6.

7. Sampaio IIC. Saúde Mental. In: RouQuayrol MZ, organizador. Epidemiologia e Saúde. Rio de Janeiro: MEDSI; 1988.

8. Kaplan HI, Sadock BJ, Grebb IA. Compêndio de PsiQuiatria: ciências do comportamento e psiquiatria clínica. Porto Alegre: Artes Médicas; 1997.

9. Bastos FIP, Castiel LD. Epidemiologia e saúde mental no campo científico contemporâneo: labirintos Que se entrecruzam? In: Amarante P, organizador. Psieuiatria e Reforma Psiquiátrica. Rio de Janeiro: FIOCRUZ; 1994.

10. Ortiz ER. Enfoque de Risco e Planejamento de Ações de Saúde. In: Rouquayrol MZ, organizador. Epidemiologia e Saúde. Rio de Janeiro: MEDSI; 1988.

11. Vilasbôas ALQ, Paim IS. Práticas de planejamento e implementação de políticas no âmbito municipal. Cad Saúde Pública 2008; 24: 1239-50. 